\title{
4th International Workshop on Semantic and Conceptual Issues in GIS (SeCoGIS 2010)
}

\section{Preface}

Recent advances in information technologies have increased the production, collection, and diffusion of geographical data, thus favoring the design and development of geographic information systems (GIS). Nowadays, GISs are emerging as a common information infrastructure, which penetrate into more and more aspects of our society. This has given rise to new methodological and data engineering challenges in order to accommodate new users' requirements for new applications. Conceptual and semantic modeling are ideal candidates to contribute to the development of the next generation of GIS solutions. They allow to elicit and capture user requirements as well as the semantics of a wide domain of applications.

The SeCoGIS workshop brings together researchers, developers, users, and practitioners carrying out research and development in geographic information systems. The aim is to stimulate discussions on the integration of conceptual modeling and semantics into current geographic information systems, and how this will benefit the end users. The workshop provides a forum for original research contributions and practical experiences of conceptual modeling and semantic web technologies for GIS, fostering interdisciplinary discussions in all aspects of these two fields, and will highlight future trends in this area. The workshop is organized in a way to highly stimulate interaction amongst the participants.

This edition of the workshop attracted papers from 11 different countries distributed all over the world: Brazil, Canada, Chile, France, Italy, Lebanon, Mexico, Spain, Switzerland, United Kingdom, and USA. We received 17 papers from which the Program Committee selected 5 papers, making an acceptance rate of $29 \%$.

The accepted papers were organized in two sessions. The first one is devoted to semantical aspects, where the first paper focuses on publishing Deep Web data, and the latter two are focused on semantic interoperability. In the second session, two papers focusing on implementation aspects will be presented.

We would like to express our gratitude to the program committee members and the external referees for their hard work in reviewing papers, the authors for submitting their papers, and the ER 2010 organizing committee for all their support. 